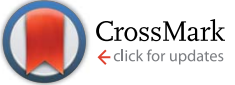

Cite this: RSC Adv., 2017, 7, 7375

Received 28th November 2016 Accepted 17th January 2017

DOI: 10.1039/c6ra27459e

www.rsc.org/advances

\section{High-purity helical carbon nanotubes with enhanced electrochemical properties for supercapacitors $\dagger$}

\begin{abstract}
Qing Zeng, ${ }^{a}$ Hanqing Tian, ${ }^{a}$ Jing Jiang, ${ }^{a}$ Xiaobo Ji, ${ }^{b}$ Daojiang Gao*c and Chao Wang ${ }^{\star a}$
The facile preparation of catalysts for high-purity helical carbon nanotubes (HCNTs) remains an open research problem. A novel catalyst precursor ferrous tartrate $\left(\mathrm{C}_{4} \mathrm{H}_{4} \mathrm{O}_{6} \mathrm{Fe}\right)$ obtained by one-step chemical synthesis is investigated in this study. The influence of reaction temperature on the morphology of the precursor's decomposition products under $\mathrm{H}_{2}$ is analyzed while Fe particles with a macroporous structure are obtained. HCNTs with a coil diameter and coil pitch of about $0.26 \mu \mathrm{m}$ and $0.28 \mu \mathrm{m}$ are achieved using $\mathrm{C}_{4} \mathrm{H}_{4} \mathrm{O}_{6} \mathrm{Fe}$ as catalyst precursor at $550{ }^{\circ} \mathrm{C}$. Interestingly, Fe particles with different crystal faces were observed. In addition, electrochemical double-layer capacitors (EDLCs) utilizing HCNTs obtained at $550{ }^{\circ} \mathrm{C}$ as electrode materials are assembled exhibiting an enhanced specific capacitance of $95 \mathrm{~F} \mathrm{~g}^{-1}$ after acid treatment at $0.1 \mathrm{~A} \mathrm{~g}^{-1}$ in $\mathrm{Na}_{2} \mathrm{SO}_{4}$.
\end{abstract}

\section{Introduction}

With the rapid development of carbon-based materials, diversiform carbon materials can be prepared, such as carbon nanotubes (CNTs), ${ }^{\mathbf{1}}$ carbon nanofibers $(\mathrm{CNF}),{ }^{2-4}$ carbon spheres, ${ }^{5}$ carbon aerogel, ${ }^{6}$ carbon nanocoils (CNC), ${ }^{7}$ graphene and so on.$^{8-13}$ Due to the unique structure and outstanding electronic properties, these carbon materials play significant roles in various fields, especially in energy storage areas such as Li-ion batteries and supercapacitors. ${ }^{14,15}$ Moreover, the discovery of a helical carbon structure has attracted increasing attentions because of its excellent elasticity and high specific surface area. Helical carbon nanotubes (HCNTs) are generally synthesized by catalytic chemical vapor deposition (CCVD) using organic substances (such as acetylene and methane) as carbon source. The morphology of this kind of carbon materials strongly depends on the properties of the catalysts and the growth temperature. ${ }^{16,17}$ To obtain high-purity HCNTs, metal catalysts have been frequently investigated and the effects of catalysts' properties on the growth of HCNTs have been analyzed. Tang et al. obtained HCNTs as the main products in the pyrolysis of acetylene at $450{ }^{\circ} \mathrm{C}$ using Fe nanoparticles as

${ }^{a}$ Clean EneElectronicrgy Materials and Engineering Center, School of Microelectronics and Solid-State Electronics, University of Electronic Science and Technology of China, Chengdu 611731, P. R. China.E-mail: cwang@uestc.edu.cn

${ }^{b}$ Key Laboratory of Resources Chemistry of Nonferrous Metals, Ministry of Education, College of Chemistry and Chemical Engineering, Central South University, Changsha 410083, China

${ }^{c}$ College of Chemistry and Materials Science, Sichuan Normal University, Chengdu, 610068, P. R. China. E-mail: daojianggao@126.com

$\dagger$ Electronic supplementary information (ESI) available. See DOI: 10.1039/c6ra27459e catalyst which was generated through a method of combined sol-gel/reduction. The results showed that the catalytic particle size played a key role in the formation of HCNTs. However, the synthetic routes to Fe nanoparticles are complex. ${ }^{17,18}$ Therefore, it is very urgent and important to develop a novel and inexpensive catalyst that can facilely prepare high-purity HCNTs.

It is well known that electrochemical double-layer capacitor (EDLC) is a kind of energy storage device with high power density, fast charge-discharge rate, low maintenance cost and long cycle life. The energy storage mechanism of EDLCs is the adsorption/desorption of the electrons on the surface of the electrode materials. ${ }^{\mathbf{1 9}}$ Hence, the specific surface area is considered as an extremely important factor for high capacitance. Thanks to the specific structure and large specific surface area, carbon materials become the main electrode materials for EDLCs. ${ }^{15,20-26}$ Carbon materials possess good electrical conductivity and are environmentally friendly, which further explains why carbon materials can be excellent electrode materials for EDLCs. At present, the specific capacitance of activated carbon can reach to $100 \mathrm{~F} \mathrm{~g}^{-1}$ with pore size of $\sim 1.4 \mathrm{~nm}$ which is difficult to be controlled. Pandolfo et al. reported that for purified nanotubes, the specific capacitance varies typically from 15 to $80 \mathrm{~F} \mathrm{~g}^{-1}$ with surface areas that range from $\sim 120$ to $400 \mathrm{~m}^{2} \mathrm{~g}^{-1} \cdot{ }^{15}$ For better electrochemical properties and larger capacitance, extensive attention has been put on the helical carbon materials due to its unique structure, morphology and large specific surface area. $\mathrm{Wu}$ et al. proved that the specific surface area was increased largely due to the helical carbon structure which was better for carbon materials in the application of supercapacitors. ${ }^{27}$

Although many achievements have been obtained in this field, there are some aspects that still need further study on the 
preparation and electrochemical performances of HCNTs, including how to synthesize high-purity carbon nanotubes via a facile and environment friendly process, how to effectively improve the electrochemical performances of HCNTs in supercapacitors and so on.

In the present study, we develop a facile and environment friendly route to synthesize high-purity HCNTs using $\mathrm{C}_{4} \mathrm{H}_{4} \mathrm{O}_{6} \mathrm{Fe}$ as catalyst precursor, which is obtained by one-step chemical synthesis. Especially, the influences of the decomposition temperature on the morphology and catalytic properties of the in situ synthesized Fe particles are discussed in detail. Moreover, the as-synthesized HCNTs are activated by the mixture of $\mathrm{H}_{2} \mathrm{SO}_{4}(98 \%)$ and $\mathrm{HNO}_{3}(98 \%)$ and assembled into EDLCs, and their electrochemical performances also are investigated..$^{28}$ Our study provides significant insights into the researches and applications on CNTs.

\section{Experimental details}

\subsection{Preparation of catalyst precursor}

All chemicals for preparation of $\mathrm{C}_{4} \mathrm{H}_{4} \mathrm{O}_{6} \mathrm{Fe}$ were purchased from KESHI and analytical reagent grade. To prepare $\mathrm{C}_{4} \mathrm{H}_{4} \mathrm{O}_{6} \mathrm{Fe}, 100$ $\mathrm{mL}$ of $1 \mathrm{M} \mathrm{FeCl}_{2}$ was dropped into $100 \mathrm{~mL}$ of $1 \mathrm{M} \mathrm{C}_{4} \mathrm{H}_{4} \mathrm{O}_{6} \mathrm{KNa}$ under stirring. The mixture turned to light yellow and precipitate formed was filtered after several minutes. Then the precipitate was washed with deionized water till $\mathrm{pH}$ of the solution was 7. In order to remove residual organic impurities, the obtained product was washed in soxhlet extractor with ethanol for $3 \mathrm{~h}$ and dried in vacuum oven at $90{ }^{\circ} \mathrm{C}$ for $3 \mathrm{~h}$.

To investigate the morphology of the catalyst precursor during catalytic process, $0.1 \mathrm{~g}$ of $\mathrm{C}_{4} \mathrm{H}_{4} \mathrm{O}_{6} \mathrm{Fe}$ powder was dispersed on a quartz boat which was put in the middle of the horizontal reaction tube inside a tubular furnace at room temperature and then heated up to reaction temperature $\left(400{ }^{\circ} \mathrm{C}, 450{ }^{\circ} \mathrm{C}, 500{ }^{\circ} \mathrm{C}, 550{ }^{\circ} \mathrm{C}, 600{ }^{\circ} \mathrm{C}\right.$ and $\left.700{ }^{\circ} \mathrm{C}\right)$ under $\mathrm{H}_{2}$

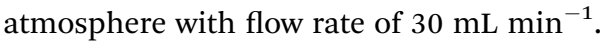

\subsection{Preparation of HCNTs and purified HCNTs}

A typical procedure of HCNTs growth is as follows. Firstly, $0.2 \mathrm{~g}$ of the as-prepared catalyst precursor was dispersed on a quartz boat, which was transferred to the horizontal reaction tube located inside a tubular furnace at room temperature. Secondly, the horizontal reaction tube was heated up to the predetermined synthesis temperature $\left(400{ }^{\circ} \mathrm{C}, 450{ }^{\circ} \mathrm{C}, 500{ }^{\circ} \mathrm{C}\right.$, $550{ }^{\circ} \mathrm{C}, 600{ }^{\circ} \mathrm{C}$ and $700{ }^{\circ} \mathrm{C}$ ) under $\mathrm{H}_{2}$ atmosphere with flow rate

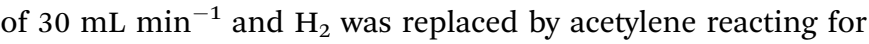
$1 \mathrm{~h}$ to grow HCNTs when the temperature was stable. Finally, the samples were naturally cooled down to room temperature under $\mathrm{N}_{2}$ atmosphere.

As for the posttreatment, HCNTs (500 mg) prepared at $550{ }^{\circ} \mathrm{C}$ were added into $40 \mathrm{~mL}$ mixed acid $\left(30 \mathrm{~mL} \mathrm{H}_{2} \mathrm{SO}_{4}(98 \%)\right.$ and 10 $\left.\mathrm{mL} \mathrm{HNO}_{3}(98 \%)\right)$. Then the mixture was sonicated for $5 \mathrm{~h}$ and diluted for next process. ${ }^{22}$ The obtained precipitation was centrifuged and washed with deionized water several times, and then dried at $60{ }^{\circ} \mathrm{C}$ in vacuum oven for $24 \mathrm{~h} .{ }^{29}$

\subsection{Preparation of electrode and electrochemical measurement}

The electrode slurry were prepared by mixing 80 wt $\%$ asprepared sample obtained at $550{ }^{\circ} \mathrm{C}, 10 \mathrm{wt} \%$ super $\mathrm{P}$ carbon black and $10 \mathrm{wt} \%$ polyvinylidene fluoride (PVDF) in $N$-methyl-2pyrrolidone (NMP) and then stirred for several hours. ${ }^{30,31}$ And the obtained slurry was casted onto carbon cloth with area of $1 \mathrm{~cm}$ by $1 \mathrm{~cm}$ and dried at $60{ }^{\circ} \mathrm{C}$ in vacuum oven. ${ }^{32}$ The mass of the active material was about $2 \mathrm{mg}$. Here, all chemicals used for preparation of slurry were purchased from Power Battery Material Co. Ltd and of battery grade. The electrochemical performances were studied by three-electrode configuration using Pt sheet as counter electrode, SCE electrode as reference electrode and active materials as working electrode. $1 \mathrm{M} \mathrm{Na}_{2} \mathrm{SO}_{4}$ (KESHI) aqueous solution was used as electrolyte. ${ }^{33}$ As for specific capacitance, it can be calculated using $C=I \Delta t / m \Delta V$ (eqn (1)) based on galvanostatic charge/discharge curves, where $I$ is the discharging current, $\Delta t$ is the discharging time, $m$ is the mass of active material and $\Delta V$ is the voltage window during discharging. ${ }^{34,35}$

\subsection{Instruments}

In the experiments, centrifugal separator (TL80-1, Tianli, Jiangsu) was used to centrifuge the precipitate and ultrasonic dispersion (KH2200E, Hechuang, Kunshan) was used for purifying HCNTs. And HCNTs was obtained by using tubular furnace (SX-G03123K, Zhonghuan, Tianjin). Vacuum oven (DZF6020 , Kejin, Hefei) was used for drying the electrode. The electrochemical properties were characterized by electrochemical workstation (P4000, Princeton, USA). The X-ray diffraction (XRD) patterns were obtained on an X-ray powder diffractometer (XRD-7000X), and field emission scanning electron microscopy (FESEM, JSM-7600F) and transmission electron microscope (TEM, JEOL-2010) were used to observe the morphology and size of products.

\section{Results and discussion}

Carbon nanomaterial and its hybrid are novel functional materials drawing great attentions. At present, two main methods including "catalyst-component" adjustment and "growth-condition" processes are used to obtain HCNTs. To obtain HCNTs, we choose $\mathrm{C}_{4} \mathrm{H}_{4} \mathrm{O}_{6} \mathrm{Fe}$ as catalyst precursor and design the unique morphology of catalyst and reasonable growing condition as shown in Fig. 1. The decomposing products of $\mathrm{C}_{4} \mathrm{H}_{4} \mathrm{O}_{6} \mathrm{Fe}$ perform different morphology under $\mathrm{H}_{2}$ atmosphere at different temperatures. Interestingly, when the temperature goes up to $600{ }^{\circ} \mathrm{C}$, the decomposing product forms macroporous structure. At lower temperature of $550{ }^{\circ} \mathrm{C}$, $\mathrm{C}_{4} \mathrm{H}_{4} \mathrm{O}_{6} \mathrm{Fe}$ is completely decomposed into Fe particle (shown in Fig. 5), then HCNTs grow with high purity by introducing $\mathrm{C}_{2} \mathrm{H}_{2}$ at the flow rate of $30 \mathrm{~mL} \mathrm{~min}{ }^{-1}$. The main reason of the helical carbon structure is resulted from the different catalytic activity and catalytic anisotropic property of the decomposing products. Herein, we offer a facile approach to obtain macroporous Fe particles and effective way to grow HCNTs. 


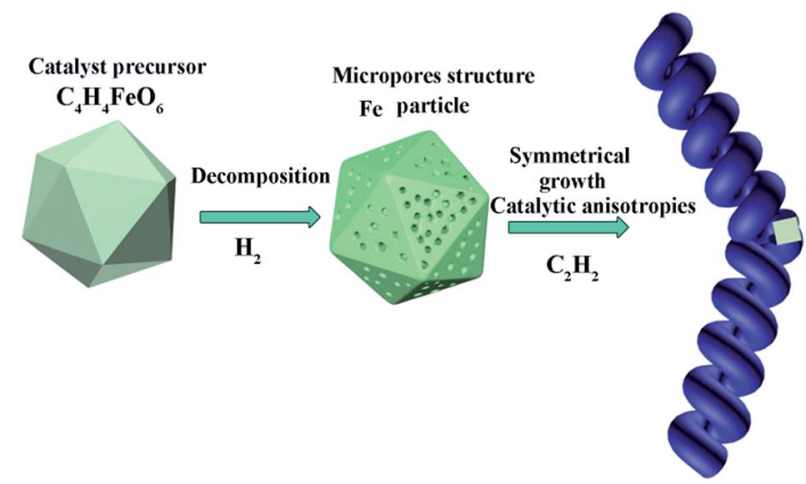

Fig. 1 Scheme of mesoporous Fe particles and the selective growth for the HCNTs using $\mathrm{C}_{4} \mathrm{H}_{4} \mathrm{O}_{6} \mathrm{Fe}$ as catalyst precursor and $\mathrm{C}_{2} \mathrm{H}_{2}$ as carbon source by adjusting reaction temperature.

\subsection{Characterization of the catalyst}

Fig. 2 shows the morphology of the products obtained from the decomposition of $\mathrm{C}_{4} \mathrm{H}_{4} \mathrm{O}_{6} \mathrm{Fe}$ under $\mathrm{H}_{2}$ with flow rate of $30 \mathrm{~mL}$ $\min ^{-1}$ at the temperatures of $400-700{ }^{\circ} \mathrm{C}$ for $1 \mathrm{~h}$. It is found that the reaction temperature greatly affects the morphology of the as-prepared samples. Rod-like products with a vast variation in length and width, and spherical particles with relative homogeneous diameters of about $24 \mu \mathrm{m}$ are formed under the temperature of $400{ }^{\circ} \mathrm{C}$ (Fig. 2a). The length and width of these rod-like products are about 10-120 $\mu \mathrm{m}$ and 3-24 $\mu \mathrm{m}$, respectively. Inset in Fig. 2a shows a magnified image of the surface on the products, where large quantity of spots with diameter of about $0.27 \mu \mathrm{m}$ can be observed. When the temperature is raised to $450{ }^{\circ} \mathrm{C}$, as shown in the Fig. $2 \mathrm{~b}-\mathrm{d}$, many small particles can be observed on the surface of $\mathrm{C}_{4} \mathrm{H}_{4} \mathrm{O}_{6} \mathrm{Fe}$ particles. At the temperature range of $450-550{ }^{\circ} \mathrm{C}$, the small particles grow with the increasing temperature. It is interesting that by raising temperature further to $600{ }^{\circ} \mathrm{C}$, numerous pores with the sizes range of 70-600 $\mathrm{nm}$ are formed (Fig. 2e). At the same time, recrystallization happened in the fine grains contributes to the smoothness of the particle surface. ${ }^{36}$ However, when temperature reached to $700{ }^{\circ} \mathrm{C}$, the pores grows larger and every large aggregation forms the petals which are shown in the Fig. $2 f$ where inset shows the magnified image.

At the temperature of $400{ }^{\circ} \mathrm{C}$, the precursor particles are still in the forms of block. Hence, it is suggested that the decomposition of the $\mathrm{C}_{4} \mathrm{H}_{4} \mathrm{O}_{6} \mathrm{Fe}$ particles are not completely finished. ${ }^{37}$ With the increase of temperature, the products from decomposition gradually form into Fe particles and recrystallization phenomenon will happen. It is considered that escaping channels for gas from decomposition reaction inside precursor form the macropores at the high temperature $\left(>550{ }^{\circ} \mathrm{C}\right)$. Furthermore, higher temperature contributes to more violent decomposition reaction of catalyst precursor and more recrystallizations on the surfaces, which results in larger pores, as explained by the Fig. 2 .

In the typical XRD pattern of product decomposing from $\mathrm{C}_{4} \mathrm{H}_{4} \mathrm{O}_{6} \mathrm{Fe}$ at $600{ }^{\circ} \mathrm{C}$ under $\mathrm{H}_{2}$ presented in Fig. 3, the three characteristic diffraction peaks of metallic ferrite over $40^{\circ}$ are clearly observed, which agree well with the standard ferrite

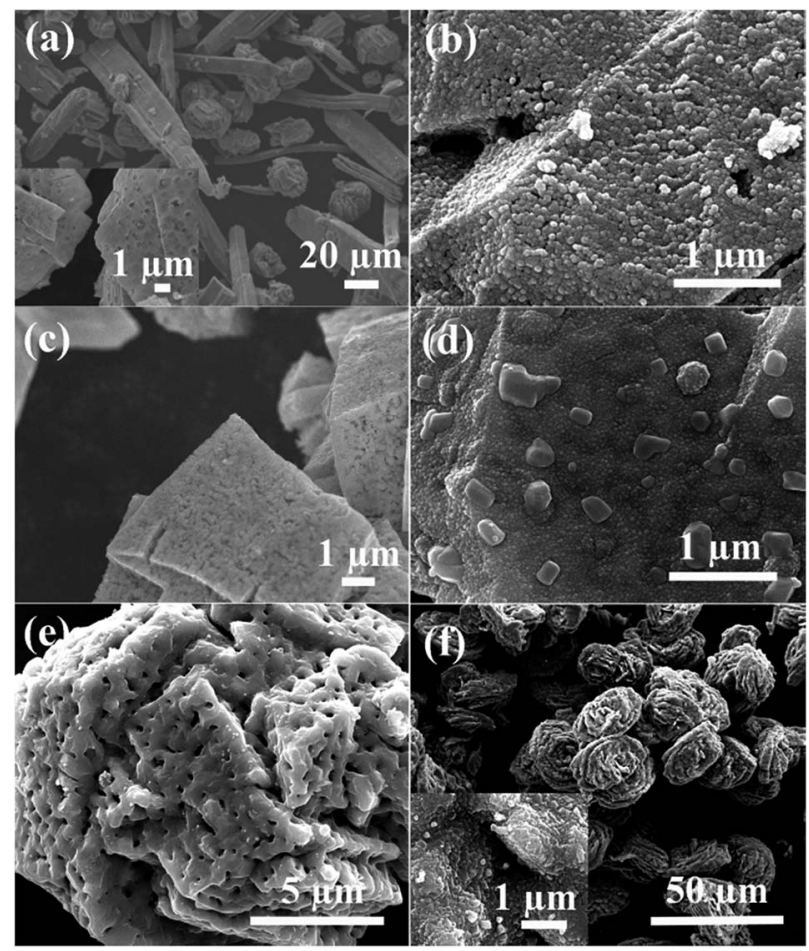

Fig. 2 SEM images of products obtained by $\mathrm{C}_{4} \mathrm{H}_{4} \mathrm{O}_{6} \mathrm{Fe}$ decomposing under $\mathrm{H}_{2}$ at the temperature of (a) $400{ }^{\circ} \mathrm{C}$, (b) $450{ }^{\circ} \mathrm{C}$, (c) $500{ }^{\circ} \mathrm{C}$, (d) $550{ }^{\circ} \mathrm{C}$, (e) $600^{\circ} \mathrm{C}$ and (f) $700^{\circ} \mathrm{C}$.

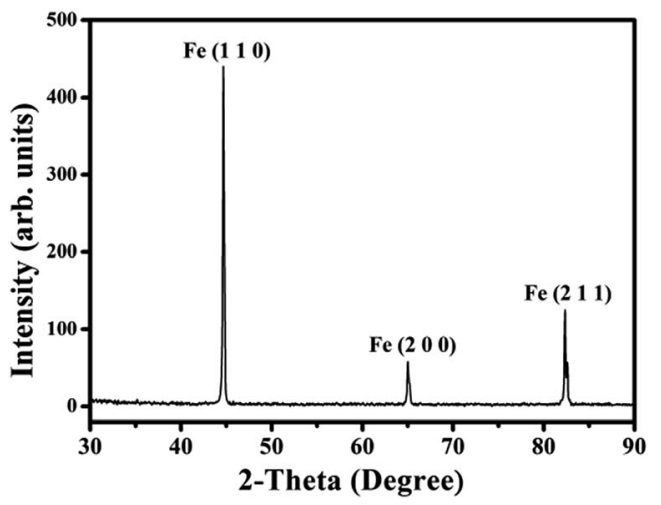

Fig. 3 XRD pattern of product after the decomposition of the $\mathrm{C}_{4} \mathrm{H}_{4} \mathrm{O}_{6} \mathrm{Fe}$ at the temperature of $600{ }^{\circ} \mathrm{C}$.

diffraction pattern (ICDD, PDF file no. 00-006-0696). Three peaks centered at $44.6^{\circ}, 65.0^{\circ}$ and $82.3^{\circ}$ correspond to the (110), (200) and (211) crystal planes, respectively. No diffraction peak of other metallic impurity is noted in the experiments. The average grain size of the catalyst particle is estimated about $57 \mathrm{~nm}$ by the Scherrer formula using the full width at half maximum value of the XRD diffraction peaks. ${ }^{38}$

\subsection{Microstructures of HCNTs}

Fig. 4 shows the SEM images of the carbon products obtained by using $\mathrm{C}_{4} \mathrm{H}_{4} \mathrm{O}_{6} \mathrm{Fe}$ as precursor in the pyrolysis of acetylene at temperatures of $400-700{ }^{\circ} \mathrm{C}$. When the temperature is under 


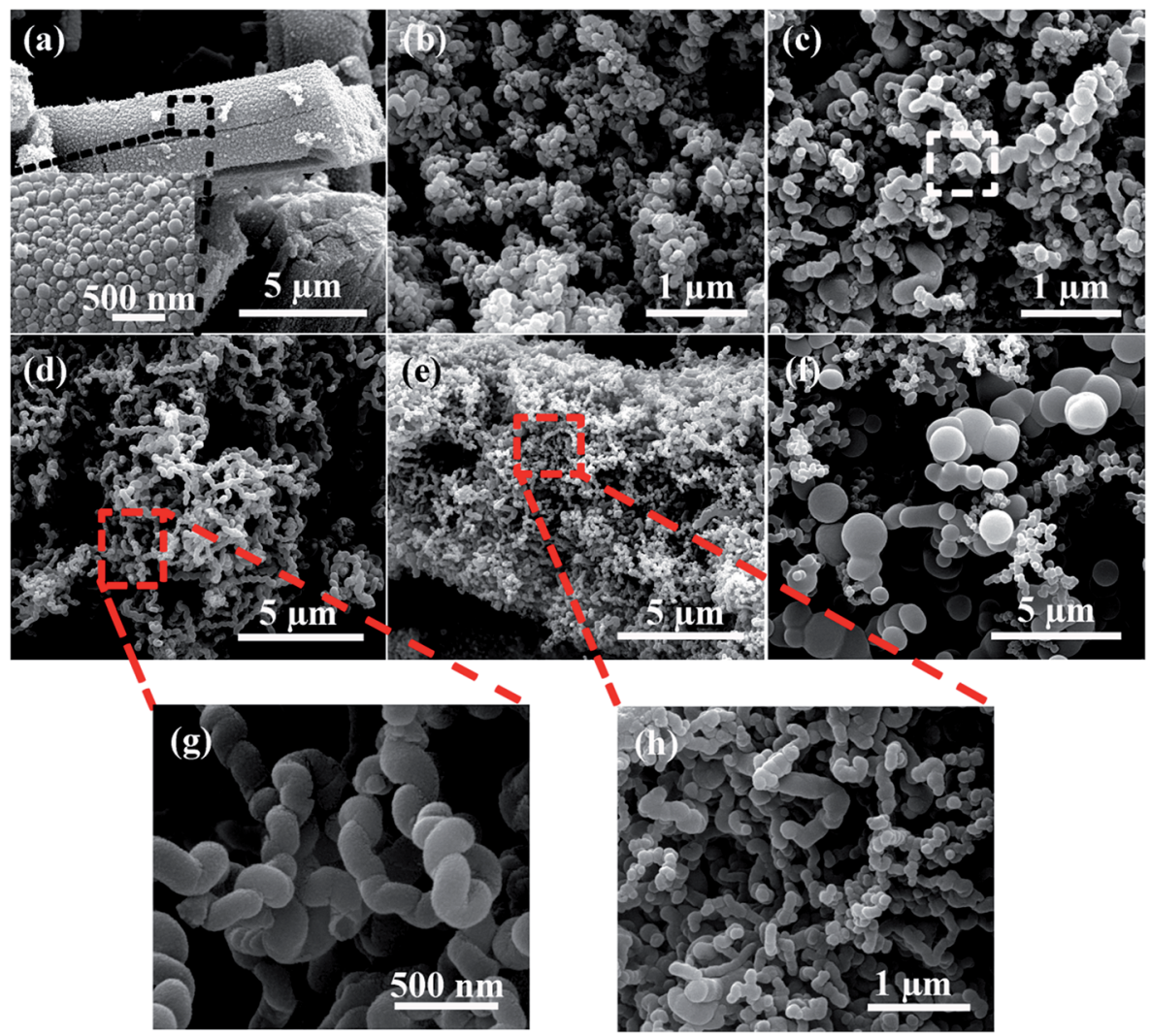

Fig. $4 \mathrm{SEM}$ images of the carbon products obtained at the growth temperatures of (a) $400{ }^{\circ} \mathrm{C}$, (b) $450{ }^{\circ} \mathrm{C}$, (c) $500{ }^{\circ} \mathrm{C}$, (d) $550{ }^{\circ} \mathrm{C}$, (e) $600{ }^{\circ} \mathrm{C}$, (f) $700^{\circ} \mathrm{C}$. Inset of (a) and red rectangles with higher magnified images ( $\mathrm{g}$ and h) marked in (d) and (e) show their partially enlarged images.

$450{ }^{\circ} \mathrm{C}$, the catalyst precursor decomposes incompletely, as shown in the Fig. 2a and b. Inset of the Fig. $4 \mathrm{a}$ is the magnified image of the product prepared at $400{ }^{\circ} \mathrm{C}$ and Fig. $4 \mathrm{~b}$ shows the pattern of the product prepared at $450{ }^{\circ} \mathrm{C}$, which agree well with the above discussion of precursor decomposing process. The particle size of Fig. $4 \mathrm{a}$ is at the range of $29-165 \mathrm{~nm}$. As the temperature is raised to $500{ }^{\circ} \mathrm{C}$, the HCNTs with sprout-like shape start to grow on the surface of the catalyst particles generated from the decomposition of the catalyst precursor. The hollow structure marked by the dashed rectangle in the Fig. 4c of the carbon nanotubes, can be observed clearly. The HCNTs with average coil diameter of $0.2 \mu \mathrm{m}$ and coil pitch of $0.19 \mu \mathrm{m}$ have the average length about $1 \mu \mathrm{m}$. Fig. $4 \mathrm{~d}$ shows the SEM image of the HCNTs obtained at $550{ }^{\circ} \mathrm{C}$, which exhibits the homogeneous coil diameter of $0.26 \mu \mathrm{m}$ and coil pitch of 0.28 $\mu \mathrm{m}$. The average length and diameter are about $5 \mu \mathrm{m}$ and 0.14 $\mu \mathrm{m}$, respectively. More importantly, the purity of the carbon product can reach to $98 \%$. The magnified image in Fig. 4e shows that carbon nanotube with zigzag morphology grows on the surface of the catalyst particles under the temperature of $600{ }^{\circ} \mathrm{C}$. Besides, the length of these carbon sticks is about 0.55 $\mu \mathrm{m}$. It is suggested that higher temperature results in faster speed of reaction leading to form the coiled morphology hardly. With the further increase of temperature to $700{ }^{\circ} \mathrm{C}$, as shown in Fig. 4f, the serious aggregation of the carbon spheres (with diameters of 0.4-1.7 $\mu \mathrm{m}$ ) and carbon particles (with smaller diameters of about $0.2 \mu \mathrm{m}$ ) can be observed. In a previous report about carbon spheres, Jin et al. obtained large-scale production of pure carbon spheres, with diameters from $50 \mathrm{~nm}$ to $1 \mu \mathrm{m}$, via direct pyrolysis of hydrocarbons at high temperature of 900$1200{ }^{\circ} \mathrm{C}^{39}$ The results indicated that carbon sphere can be formed from hydrocarbon at high temperature. However, Miao et al. obtained carbon spheres with diameters between 0.4 and 2 $\mu \mathrm{m}$ using kaolin supported transition metal salts (Fe, Co, Ni) as catalysts at relatively high temperature about $650{ }^{\circ} \mathrm{C}$ from acetylene. ${ }^{5}$ Acetylene is activated by metallic particles and reduced initially to form amorphous carbon. Hence, it can indicate that carbon spheres with various diameters (Fig. 4f) are obtained by the decomposition of acetylene at relatively high temperature above $650{ }^{\circ} \mathrm{C}$ with $\mathrm{Fe}$ particles as catalysts and enough reaction time.

TEM images of typical HCNTs prepared at $550{ }^{\circ} \mathrm{C}$ are shown in Fig. 5. The as-prepared HCNTs have coil diameter and coil pitch of about $300 \mathrm{~nm}$, which is in accordance with SEM images. The inside catalyst particles are polyhedral shape with a diameter of 80-260 $\mathrm{nm}$. In addition, most of these facet particles have different length in edge without symmetry in architecture, wrapped in the middle of the helical carbon nanotubes, as shown in Fig. 5b. The partial part with helical structure of the HCNTs can be clearly observed in Fig. 5c. Inset of Fig. 5c above with higher magnified image exhibits two overlapped, reverse crystal planes forming the helical structure. It indicates that the nanotubes are composed of unclosed quasi-graphene layers with the interlayer distances at the range of about 3.3-3.5 $\AA$, 


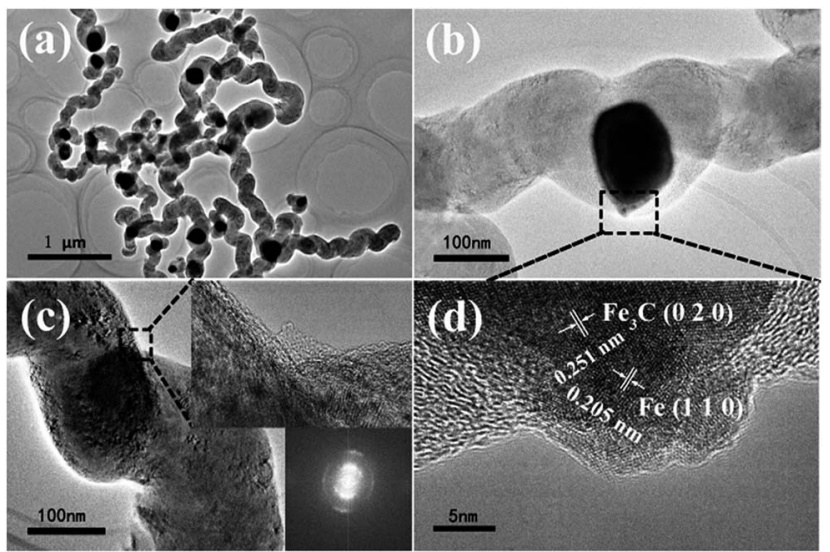

Fig. 5 TEM images of (a) typical HCNTs obtained at $550{ }^{\circ} \mathrm{C}$, (b) HCNT with catalyst, (c) helical structure of HCNT and (d) magnified image of marked part in (b). Insets of (c) are magnified image of the zigzag area and its FFT pattern.

which are consistent with the results reported by Wang et al. ${ }^{39,40}$ From the Fast Fourier Transform (FFT) pattern inset in Fig. 5c, it is suggested that HCNT is a kind of entangled semicrystalline structure. Fig. $5 \mathrm{~d}$ is a magnified image of the dashed rectangle marked part in Fig. 5b. The edge area of catalyst particle covered by carbon layers is further investigated in order to figure out the crystal phase. A bulge exists on the surface of catalyst particle, which is suggested to be the active site for HCNTs growth. ${ }^{3}$ Meanwhile, the FFT pattern contains the diffraction patterns of monocrystal grain and polycrystal grain. Two kinds of crystal planes are observed with plane distance of about $2.0 \AA$ and 2.5 $\AA$, respectively. The alignments and the interlayer distance of these crystal planes agree well with $\mathrm{Fe}(110)$ and $\mathrm{Fe}_{3} \mathrm{C}(020)$ (ICDD, PDF file no. 00-003-0400) planes. ${ }^{41}$ The results confirm that the dark zone wrapping catalyst particle in middle of CNT is a kind of $\mathrm{Fe}^{-} \mathrm{Fe}_{3} \mathrm{C}-\mathrm{CNT}$ hybrid. ${ }^{42,43}$

XRD patterns of HCNT products obtained at $450{ }^{\circ} \mathrm{C}, 550{ }^{\circ} \mathrm{C}$ and $600{ }^{\circ} \mathrm{C}$ are shown in Fig. S1. $\dagger$ The diffraction peaks can be indexed to the phases of graphene, $\mathrm{Fe}$ and $\mathrm{Fe}_{3} \mathrm{C}$, which in accordance to the results of $\mathrm{Fe}-\mathrm{Fe}_{3} \mathrm{C}-\mathrm{CNT}$ hybrid discussed above. ${ }^{44,45}$

\subsection{Electrochemical behaviour of HCNTs}

Since the change of Brunauer-Emmett-Teller $\left(\operatorname{BET}\left(\mathrm{N}_{2}\right)\right)$ surface area and the pore size distribution are correlated directly to the specific capacitance, the nitrogen adsorption/desorption isotherms (Fig. S3†) and pore size distribution (Fig. S4†) have been conducted to further confirm the texture properties of pristine HCNTs and a-HCNTs. The BET surface area enhances from $16.6 \mathrm{~m}^{2} \mathrm{~g}^{-1}$ of HCNTs to $19.3 \mathrm{~m}^{2} \mathrm{~g}^{-1}$ of a-HCNTs. The pore size distribution is mainly at range of $5-50 \mathrm{~nm}$, indicating the mesopores. The mesopore volume of a-HCNTs is obviously larger than HCNTs suggesting better electrochemical performance of a-HCNTs, which is verified in the next paragraphs.

The electrochemical properties, especially the specific capacitance and equivalent series resistance (ESR), are examined by standard cyclic voltammetry (CV), galvanostatic charge/ discharge technique (GCD) and electrochemical impedance spectroscopy (EIS). ${ }^{29,46}$ The corresponding CV, GCD and EIS profiles of HCNTs are shown in Fig. S5. $\dagger$ The HCNTs electrode exhibits nearly in rectangular shape, even at high scanning rate of $10 \mathrm{~V} \mathrm{~s}^{-1}$, indicating a great supercapacitor behavior. The CV results also suggest that the influence of precursor existed in the HCNTs was negligible. The CV curves of HCNTs electrode with acid treatment (a-HCNTs) at different scanning rates suggest that the shape of the curves are definitively changed. A pair of remarkable reversible pseudofaradaic reactions of a-HCNTs can be obviously observed near $0.1 \mathrm{~V}$ in the $\mathrm{CV}$ curves of pristine HCNTs and a-HCNTs electrodes at scanning rate of $0.1 \mathrm{~V} \mathrm{~s}^{-1}$. It is reported by Frackowiak et al. that the HCNTs after acid treatment can maintain the initial shape and microtexture, which is confirmed by the results shown in Fig. S2..$^{23}$ However, the number of groups on the surface will increase. ${ }^{47}$ And the following redox reactions of the surface group during potentiodynamic cycling can be considered:

$$
\begin{gathered}
>\mathrm{C}-\mathrm{OH} \Leftrightarrow \supset \mathrm{C}=\mathrm{O}+\mathrm{H}^{+}+\mathrm{e}^{-} \\
\succ \mathrm{C}=\mathrm{O}+\mathrm{e}^{-} \Leftrightarrow \supset \mathrm{C}^{-} \mathrm{O}^{-}
\end{gathered}
$$

Moreover, pseudocapacitance could gradually decrease after long cycling due to the quasireversible reactions. In the $\mathrm{CV}$ curves, the current responding of a-HCNTs is tremendously higher than the pristine HCNTs at the same scanning rate, which means an extraordinarily higher capacitance than that of pristine HCNTs. ${ }^{22}$

In order to further evaluate the electrochemical performance of the HCNTs, charge-discharge cycling curves within the potential window from $-0.2 \mathrm{~V}$ to $0.8 \mathrm{~V}$ were analyzed (Fig. S5d $\dagger$ ). ${ }^{22}$ The curve of HCNTs is nearly linear indicating that the electrode works in the double layer capacitance mode mainly. However, the curve of a-HCNTs has reduction peak which suggests that the electrode has reduction reaction. The result is consistent with $\mathrm{CV}$ curve discussed above and further testifies the existence of "Pesudo" region resulted from the surface group. ${ }^{48}$ The specific capacitance of the pristine HCNTs and a-HCNTs calculated on the base of the discharge curves using eqn (1) are about $1 \mathrm{~F} \mathrm{~g}^{-1}$ and $95 \mathrm{~F} \mathrm{~g}^{-1}$ respectively at current density of $0.1 \mathrm{~A} \mathrm{~g}^{-1}$. Obviously, the posttreatment is powerful factor in increasing the specific capacitance of HCNTs. The specific capacitances for HCNTs based on the discharge curves are about $95,78,73$ and $68 \mathrm{~F} \mathrm{~g}^{-1}$ at $0.1,0.3,0.5$ and $1 \mathrm{~A}$ $\mathrm{g}^{-1}$, respectively (Fig. S5e $\dagger$ ). It is suggested that a-HCNTs has good capacitance performance even at high current density of 1 $\mathrm{A} \mathrm{g}^{-1}$.

The EIS measurement is another important technique to analyze the charge storage mechanism. The curve is divided into two different parts including linear region at low frequency and semicircle region at high frequency (Fig. S5f $\dagger$ ). The line representing for low frequency region is related to the electrode material conductivity, ion diffusion between electrolyte and electrode material. And the semicircle at high frequency region is connected to contact resistance between electrode material 
and current collector and charge transfer resistance. ${ }^{30}$ It is possible to estimate the ESR which correlates to the value of $X$ intercept of the semicircle with $Z^{\prime}$ axis based on the Nyquist plot. The ESR of a-HCNTs is about $3.9 \Omega$ evaluated from the EIS curve where $Z^{\prime \prime}$ is zero. The low ESR is due to high conductivity and good contact with the carbon cloth as current collector. Meanwhile, cycling stability of a-HCNTs electrode has been examined (Fig. S6 $\dagger$ ). When as-sample is tested at current of $0.1 \mathrm{~A}$ $\mathrm{g}^{-1}$, the discharge capacity and coulombic efficiency are obtained as $50 \mathrm{~F} \mathrm{~g}^{-1}$ and $100 \%$ after 1000 cycles. All above discussions show that the electrochemical performance of HCNTs after acid treatment is enhanced a lot as electrode material used in the supercapacitors.

\section{Conclusions}

In conclusion, HCNTs, with coil diameter of about $0.26 \mu \mathrm{m}$ and pitch of about $0.28 \mu \mathrm{m}$ were obtained from acetylene by using $\mathrm{C}_{4} \mathrm{H}_{4} \mathrm{O}_{6} \mathrm{Fe}$ as catalyst precursor at $550{ }^{\circ} \mathrm{C}$, with the high purity of $98 \%$. The influence of temperature at $400-700{ }^{\circ} \mathrm{C}$ on the catalyst precursor morphology under $\mathrm{H}_{2}$ atmosphere was investigated. Moreover, microporous structure was formed with the temperature increasing and the obtained products were Fe particles with different crystal faces under the decomposition completing. SEM images of HCNTs results and XRD of catalyst confirmed that helical structure arise from different crystal faces with different catalytic activities. Since carbon based materials played important roles in the application on EDLCs, as-prepared HCNTs after acid treatment showed excellent electrochemical properties. HCNTs as electrode materials exhibited regular rectangle shape even at high scanning rate. And the specific capacitance of $95 \mathrm{~F} \mathrm{~g}^{-1}$ was obtained at current density of $0.1 \mathrm{~A} \mathrm{~g}^{-1}$. With further post treatment, HCNTs could exhibit even better electrochemical properties.

\section{Acknowledgements}

This work was supported by the open fund of State Key Laboratory of Electronic Thin Films and Integrated Devices of UESTC (No. KF14008), State Key Laboratory of Advanced Welding and Joining of HIT (No. AWJ-M16-07), the Fundamental Research Funds for the Central Universities of UESTC (No. ZYGX2013J115, ZYGX2014J087, ZYGX2015J029), and the Project of the Science and Technology Department in Sichuan Province Supporting Plan (No. 2014GZ0151, 2016JQ0022).

\section{Notes and references}

1 E. V. Shlyakhova, N. F. Yudanov, Y. V. Shubin, L. I. Yudanova, L. G. Bulusheva and A. V. Okotrub, Carbon, 2009, 47, 17011707.

2 X. Jian, Z. Zhou, S. Wu, L. Chen, Q. Zeng and C. Wang, Nanoscale Res. Lett., 2014, 9, 1-7.

3 X. Jian, M. Jiang, Z. Zhou, M. Yang, J. Lu, S. Hu, Y. Wang and D. Hui, Carbon, 2010, 48, 4535-4541.

4 M.-Q. Wang, C. Ye, S.-J. Bao, Z.-Y. Chen, Y.-N. Yu, Y. Zhang and M.-W. Xu, Chem. Commun., 2016, 52, 12992-12995.
5 J.-Y. Miao, D. W. Hwang, K. V. Narasimhulu, P.-I. Lin, Y.-T. Chen, S.-H. Lin and L.-P. Hwang, Carbon, 2004, 42, 813-822.

6 R. Saliger, U. Fischer, C. Herta and J. Fricke, J. Non-Cryst. Solids, 1998, 225, 81-85.

7 X. Jian, X. Chen, Z. Zhou, G. Li, M. Jiang, X. Xu, J. Lu, Q. Li, Y. Wang and J. Gou, Phys. Chem. Chem. Phys., 2015, 17, 30243031.

8 D. Li, L. Pan, Y. Wu and W. Peng, Carbon, 2012, 50, 25712580.

9 X. Ren, H. Zhang and Z. Cui, Mater. Res. Bull., 2007, 42, 22022210.

10 D. Sarangi and A. Karimi, Nanotechnology, 2003, 14, 109.

11 J.-B. Park, G.-S. Choi, Y.-S. Cho, S.-Y. Hong, D. Kim, S.-Y. Choi, J.-H. Lee and K.-I. Cho, J. Cryst. Growth, 2002, 244, 211-217.

12 Q. Zhang and Z. Cui, Mater. Lett., 2009, 63, 850-851.

13 A. Dourani, M. Hamadanian, M. Haghgoo, M. R. Jahannama and H. Goudarzi, RSC Adv. , 2015, 5, 49944-49952.

14 H. Luo, K. Huang, B. Sun and J. Zhong, Electrochim. Acta, 2014, 149, 11-17.

15 A. G. Pandolfo and A. F. Hollenkamp, J. Power Sources, 2006, 157, 11-27.

16 X. Jian, M. Jiang, Z. Zhou, Q. Zeng, J. Lu, D. Wang, J. Zhu, J. Gou, Y. Wang, D. Hui and M. Yang, ACS Nano, 2012, 6, 8611-8619.

17 N. Tang, J. Wen, Y. Zhang, F. Liu, K. Lin and Y. Du, ACS Nano, 2010, 4, 241-250.

18 N. J. Tang, W. Zhong, C. T. Au, A. Gedanken, Y. Yang and Y. W. Du, Adv. Funct. Mater., 2007, 17, 1542-1550.

19 R. Kötz and M. Carlen, Electrochim. Acta, 2000, 45, 24832498.

20 Q. Zhao, X. Wang, C. Wu, H. Shu, J. Gao, Y. Zhang, J. Liu and H. Wang, Meet. Abstr., 2014, 308.

21 C. Niu, E. K. Sichel, R. Hoch, D. Moy and H. Tennent, Appl. Phys. Lett., 1997, 70, 1480-1482.

22 J. Chen, C. Jia and Z. Wan, Electrochim. Acta, 2014, 121, 4956.

23 E. Frackowiak, K. Metenier, V. Bertagna and F. Beguin, Appl. Phys. Lett., 2000, 77, 2421-2423.

24 L. Diederich, E. Barborini, P. Piseri, A. Podestà, P. Milani, A. Schneuwly and R. Gallay, Appl. Phys. Lett., 1999, 75, 2662-2664.

25 Q.-L. Chen, K.-H. Xue, W. Shen, F.-F. Tao, S.-Y. Yin and W. Xu, Electrochim. Acta, 2004, 49, 4157-4161.

26 X. Yun, J. Wang, L. Shen, H. Dou and X. Zhang, RSC Adv., 2015, 5, 22173-22177.

27 F.-Y. Wu, J.-H. Du, C.-G. Liu, L.-X. Li and H.-M. Cheng, New Carbon Mater., 2004, 2, 001.

28 Q. Jiang, X.-Y. Lu, Y. Zhao and Z.-L. Yu, Acta Phys.-Chim. Sin., 2004, 20, 546-549.

29 J. Chen, C. Jia and Z. Wan, Synth. Met., 2014, 189, 69-76.

30 D. Su, L. Pan, X. Fu and H. Ma, Appl. Surf. Sci., 2015, 324, 349-354.

31 E. Frackowiak and F. Béguin, Carbon, 2001, 39, 937-950.

32 Y. Yang, J. Loomis, H. Ghasemi, S. W. Lee, Y. J. Wang, Y. Cui and G. Chen, Nano Lett., 2014, 14, 6578-6583. 
33 O. V. Kharissova, B. I. Kharisov and E. G. de Casas Ortiz, RSC Adv., 2013, 3, 24812-24852.

34 K. H. An, W. S. Kim, Y. S. Park, J.-M. Moon, D. J. Bae, S. C. Lim, Y. S. Lee and Y. H. Lee, Adv. Funct. Mater., 2001, 11, 387-392.

35 Y. Y. Peng, Y. M. Liu, J. K. Chang, C. H. Wu, M. D. Ger, N. W. Pu and C. L. Chang, Carbon, 2015, 81, 347-356.

36 R. L. Schmid and J. Felsche, Thermochim. Acta, 1982, 59, 105114.

37 T. Palacios-Hernández, G. A. Hirata-Flores, O. E. ContrerasLópez, M. E. Mendoza-Sánchez, I. Valeriano-Arreola, E. González-Vergara and M. A. Méndez-Rojas, Inorg. Chim. Acta, 2012, 392, 277-282.

38 A. L. Patterson, Phys. Rev., 1939, 56, 978-982.

39 Y. Z. Jin, C. Gao, W. K. Hsu, Y. Zhu, A. Huczko, M. Bystrzejewski, M. Roe, C. Y. Lee, S. Acquah, H. Kroto and D. R. M. Walton, Carbon, 2005, 43, 1944-1953.
40 L. Wang, C. Li, F. Gu and C. Zhang, J. Alloys Compd., 2009, 473, 351-355.

41 Y. Lu, Z. Zhu and Z. Liu, Carbon, 2005, 43, 369-374.

42 W. Yang, X. Liu, X. Yue, J. Jia and S. Guo, J. Am. Chem. Soc., 2015, 137, 1436-1439.

43 J.-S. Li, S.-L. Li, Y.-J. Tang, M. Han, Z.-H. Dai, J.-C. Bao and Y.-Q. Lan, Chem. Commun., 2015, 51, 2710-2713.

44 X. Qi, W. Zhong, Y. Deng, C. Au and Y. Du, Carbon, 2010, 48, 365-376.

45 Y. Hou, T. Huang, Z. Wen, S. Mao, S. Cui and J. Chen, Adv. Energy Mater., 2014, 4, 1400337.

46 A. Leela Mohana Reddy, R. I. Jafri, N. Jha, S. Ramaprabhu and P. M. Ajayan, J. Mater. Chem., 2011, 21, 16103-16107.

47 L.-j. Wang, L.-c. Zhong, F. Gu and J.-z. Xiao, J. China Univ. Pet., 2009, 23, 4.

48 H. Pan, J. Li and Y. Feng, Nanoscale Res. Lett., 2010, 5, 654668. 